\title{
The Power of Deconvolution for Study of QSO Host Galaxies
}

\author{
Géraldine Letawe ${ }^{1}$, Yannick Letawe ${ }^{1}$, and Pierre Magain ${ }^{1}$ \\ ${ }^{1}$ Université de Liège, Allée du 6 aout 174000 Liège, Belgium \\ Email: gletawe@ulg.ac.be
}

\begin{abstract}
The major challenge in studying QSO host galaxies is to remove the QSO contribution, which often outshines the whole system. Our group has acquired the technical ability to handle such data, for images and slit spectroscopy, as well as integral field spectroscopy. We review here the major techniques developed by our team, and their latest applications. We are open for collaboration with other teams to spread the use of these powerful techniques.
\end{abstract}

Keywords. techniques: image processing, spectroscopy, galaxies: active, quasars: general

Spectroscopy: We apply the Magain, Courbin, \& Sohy (1998) deconvolution routines to the separation of the quasar and host galaxy. In addition to detailed analysis of integrated host and quasar spectra (Letawe et al. 2007), we combine the information from spatially resolved slit spectra to high-resolution imaging from HST/ACS (Letawe et al. 2008a). These studies clarify the role of interaction in bringing gas and dust to the nucleus and reveal the dominance of host galaxies containing young stellar populations and ionized gas as well as the broad nuclear $\mathrm{H} \alpha$ reflected line far from the nucleus. The peculiar source HE 0450-2958 (Magain et al. 2005) has been a focus of this work.

Integral field spectroscopy: Based on VLT IFU observations of quasars, we remove the nuclear contributions by subtracting a weighted nuclear spectrum (taken at the central position), relying on the spectral signature of the broad nuclear Balmer lines. Dynamics, BPT diagrams, and more are thus derived from the host-alone spectra. The method is fully described and applied to HE $0450-2958$ by Letawe et al. (2008b). Up-todate synthesis on this object is described by Elbaz et al. (2009).

Imaging: A large sample of bright low-redshift quasars, observed with NTT/SUSI2 through two optical filters, has been deconvolved, separating quasar and hosts (Letawe et al. 2009). A detailed analysis of morphologies, asymmetries, and gas content of the hosts shows that (i) host galaxies have on average more ionized gas than quiescent galaxies, with the discrepancy being more pronounced for ellipticals, (ii) asymmetries in ellipticals are mainly due to a shift between the position of the active nuclei and the center of the galaxy, possibly suggestive of a $\mathrm{BH}$ recoil after a binary merger, and (iii) $60 \%$ of the hosts show signs of interaction, both ellipticals ( $50 \%$ of the sample) and spirals (25\% of the sample).

\section{References}

Elbaz, D., et al. 2009, A\& $A$, in press (arXiv:0907.2923)

Letawe, G., et al. 2007, MNRAS, 378, 83

Letawe, G., Magain, P., \& Courbin, F. 2008b, A\& $A$, 480, 69

Letawe, Y., Magain, P., Letawe, G., Courbin, F., \& Hutsemékers, D. 2008a, ApJ, 679, 976

Letawe, Y., Letawe G., \& Magain, P. 2009, submitted to MNRAS

Magain, P., Courbin, F., \& Sohy, S. 1998, ApJ, 494, 472

Magain, P., et al. 2005, Nature, 437, 381 